\title{
Laser-Assisted Positron-Impact Ionization of Hydrogen Atoms
}

\author{
S. Taj, B. Manaut*, E. Hrour and M. El Idrissi \\ Université Sultan Moulay Slimane, Faculté Polydisciplinaire, \\ Equipe de Recherche en Physique Théorique et Matériaux (ERPTM), P.O. Box 523, Béni Mellal, 23000, Morocco
}

(Received May 2, 2018; revised version February 22, 2019; in final form March 14, 2019)

The theory of triple differential cross-sections, developed previously within the framework of nonrelativistic Schrödinger formalism, is extended to the relativistic treatment by using the Dirac-Volkov formalism. At high energies, the colliding particles lose their Coulomb character and the channel of the symmetric coplanar geometry is opened. In the first Born approximation, we have studied theoretically the laser-assisted relativistic ionization of hydrogen atoms by positron impact in this geometry. Triple differential cross-sections are calculated by using two approaches: (i) RPWSG to describe relativistic plane wave in the symmetric geometry, (ii) DVPWSG (Dirac-Volkov plane wave in the symmetric geometry) in which we take full account of the relativistic dressing effects, has been proposed to study the influence of laser field on the colliding particles. To check the consistency of our model, we have used, for comparison, the nonrelativistic approach: nonrelativistic plane wave in the symmetric geometry. Numerical results for the variation of relativistic triple differential cross-sections and their dependences on laser field parameters (intensity, frequency) and incident positron energy are also presented. As for the laser modifications, by summing over a very large number of exchanged photons, the laser-assisted triple differential cross-sections usually tends to approach the laser-free triple differential cross-sections, obeying the famous Kroll-Watson sum rule for positron projectile. In the absence, of any experimental data at high energies for this particular ionization process, we are not in a position to compare the present results with the experimental findings.

DOI: 10.12693/APhysPolA.136.78

PACS/topics: positron impact, ionization hydrogen atom, laser assisted, QED calculations, relativistic scattering theory

\section{Introduction}

Positron-atom collisions in the presence of a laser field have recently become of central importance in practical applications [1-3] and fundamental studies [4-6]. In particular, the ionization processes have been widely investigated in the literature by several authors [7-9]. Regarding the theoretical situation, low energy positron-impact ionization of hydrogen atom in the presence of a laser field has often been discussed in several articles [10-14]. Most of these studies have formulated explicit expressions for the laser-assisted triple differential cross-sections (TDCS) by assuming a weak laser electric field compared with the characteristic inter-atomic field and a laser frequency much lower than any of the transition frequencies of the hydrogen atom. However, to the best of our knowledge, no laser-assisted $\left(\mathrm{n} \gamma \mathrm{e}^{+} ; \mathrm{e}^{+} \mathrm{e}^{-}\right)$atomic hydrogen experiment is yet available in the relativistic regime. The absence of any experimental data adds further importance to the theoretical study of such a process. In a relativistic domain, we can mention the two theoretical investigations which have been made on the laser-assisted TDCS for ionization of atomic hydrogen by electron impact $[15,16]$. Note that the basic theoretical approaches to the calculation of positron scattering from atoms and molecules were originally developed for electron scattering and later applied to the positron case. Feynman is

*corresponding author; e-mail: b.manaut@usms.ma known for the interpretation of the negative energy field equation solutions for antiparticles. According to the so called Feynman-Stueckelberg interpretation [17] a negative energy solution of the Dirac equation corresponds to a positron which then runs apparently backwards in time. Using in CPT transforms a $T$ violation (time reversal) [18], we show that the equation of the positron is exactly the same as the one of the electron, on the condition that both the sign of the time and the momentum are changed. However the $T$ transformation seems to imply that positron having positive energy moving forward in space-time is treated as electron having negative energy and propagating backward in time [19-21].

The present paper is an extension of the recent theoretical study of relativistic ionization of hydrogen atoms by positron impact in the absence of a laser field [22]. Our theoretical study addresses the problem of relativistic ionization of a ground-state hydrogen atom by positron impact in the presence of a circularly polarized homogeneous laser field in coplanar symmetric geometry. In the circular polarization, a simplification of generalized Bessel function is possible and it leads to a full reduction to ordinary Bessel functions which are commonly used in this case. In this geometry, $\left(\mathrm{n} \gamma \mathrm{e}^{+} ; \mathrm{e}^{+} \mathrm{e}^{-}\right)$reaction of hydrogen atom with equal energy sharing by the two outgoing particles, the ejected electron does not feel the Coulomb influence of atomic target and can be described by a plane wave in the non-relativistic limit as well as in the relativistic regime. We examine the effects of a laser field on the angular distribution of ejected electron by calculating the laser-assisted TDCSs for high energy positron impact ionization, for two distinct relativistic 
theoretical models in the presence and in the absence of laser field, respectively: (i) the Dirac-Volkov plane wave in the symmetric geometry (DVPWSG) and (ii) relativistic plane wave in the symmetric geometry (RPWSG). The consistency of our results is checked in the non relativistic limit by comparison with the nonrelativistic plane wave in the symmetric geometry (NRPWSG) using the Volkov plane wave functions to describe the two particles $\mathrm{e}^{+}$and $\mathrm{e}^{-}$.

The present work is structured as follows. In Sect. 2, we present the theory of $\left(n \gamma \mathrm{e}^{+} ; \mathrm{e}^{+} \mathrm{e}^{-}\right)$reactions of hydrogen atom in the first Born approximation both in the absence and in the presence of a laser field. A detailed account is then given of the techniques we have used to evaluate the TDCS, starting by RPWSG in the absence of the laser field, before moving to the description of the relativistic regime in the presence of the laser field by DVPWSG. In Sect. 3, we present our numerical results in which we discuss the importance of the dressing effects and we study the influence of the laser parameters (intensity, frequency) and incident positron energy on the triple differential cross-section. Section 4 summarizes our conclusions. Note that in this work atomic units are used throughout. The velocity of light is $c \simeq 137$ a.u., while energy is measured in atomic units, $1 \mathrm{a} . \mathrm{u} . \simeq 27.2116 \mathrm{eV}$. The atomic unit of intensity is $3.53 \times 10^{16} \mathrm{~W} / \mathrm{cm}^{2}$ at the electrical field strength of $\epsilon=1$ a.u. For an angular frequency of $\omega=0.043 \mathrm{a} . \mathrm{u} .=1.17 \mathrm{eV}$, corresponding to the lasing transition of the Nd:YAG laser at a wavelength of $1064 \mathrm{~nm}$. Note also that the Lorentz scalar product is defined by $(A . B)=A^{\mu} B_{\mu}$ and for all $k$, "bold" style $\mathbf{k}$ is recommended for vectors.

\section{Theoretical models}

The process in the course of which $(s+l)$ photons from the external laser field are exchanged, while the ionizing positron-atom scattering takes place, can be described by the following symbolic prototype:

$$
\mathrm{e}^{+}\left(q_{i}^{\mu}\right)+\mathrm{H} \pm(l+s) \hbar k^{\mu} \longmapsto \mathrm{H}^{+}+\mathrm{e}^{-}\left(q_{B}^{\mu}\right)+\mathrm{e}^{+}\left(q_{f}^{\mu}\right),
$$

where the indices $i, f$, and $B$ stand for incident, scattered, and ejected, respectively. The quasi-momentum $q^{\mu}$ is such that

$$
q^{\mu}=p^{\mu}-\frac{\overline{a^{2}}}{2 c^{2}\left(k \cdot p_{j}\right)} k^{\mu}
$$

where $\overline{a^{2}}$ denotes the time-averaged square of the fourvector potential of the laser field. The physical significance of $q^{\mu}$ is the averaged four-momentum of the particle inside the laser field with wave four-vector $k^{\mu}$. In Eq. (1), the integers $s$ and $l$ are respectively the number of photons exchanged between the laser field and the incident, scattered positrons and between the laser field and the ejected electron during the process. Positive integer values of $s$ and $l$ correspond to photon absorption and negative integer ones to photon emission.
For the coplanar symmetric geometry described in Fig. 1, the electron and positron angles with respect to the incident beam direction were equal to each other. In this configuration, the energies selected for the scattered positron and ejected electron were also equal.

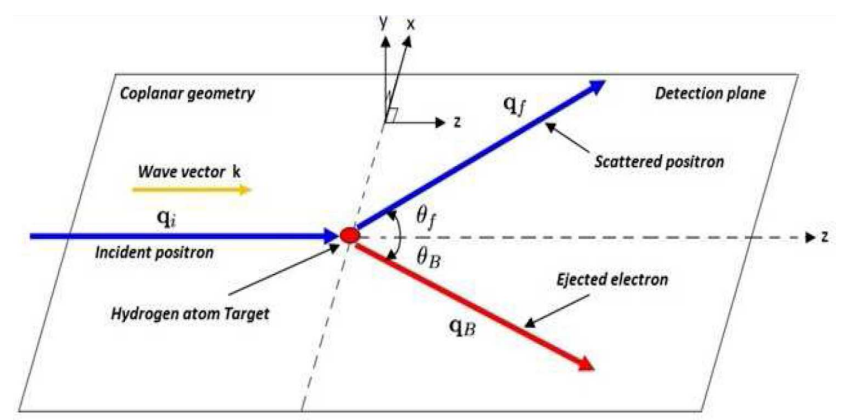

Fig. 1. The coplanar geometry chosen for the studies detailed here. $\left(\theta_{f}, \theta_{B}\right)$ are the angles of the positron and the electron with respect to the incident beam direction $\boldsymbol{q}_{i}$. For coplanar symmetric geometries, $\theta_{f}=\theta_{B}$.

In the experiments, the spin of the particles is not measured and therefore the only constraints to the kinematics of the reaction are energy and momentum conservation. This permits the outgoing positron and electron to have a range of different directions and a range of different energies. Thus, to fully describe the ionization process for a given incident energy, $E_{i}$, a TDCS is required

$$
\operatorname{TDCS}=\frac{\mathrm{d}^{3} \sigma}{\mathrm{d} E_{B} \mathrm{~d} \Omega_{B} \mathrm{~d} \Omega_{i}},
$$

where $d \Omega_{B}$ and $d \Omega_{i}$ correspond to solid angles defined by the direction of the outgoing positron and electron and $E_{B}$ represents the energy of the ejected electron. It is only necessary for one energy to be defined since the energies of the two outgoing electron and positron are related by the energy conservation. The energy conservation equation corresponding to the laser-assisted reaction (1), during which $s+l$ photons are transferred between the positron-atom system and the field is

$$
Q_{f}=Q_{i}+(s+l) \omega+\xi_{b}-Q_{B},
$$

where $\xi_{b}$ is the binding energy of the atomic electron.

We start from the first Born ionization $S$-matrix element, which is given by

$$
\begin{aligned}
& S_{f i}= \\
& \quad \frac{i}{c} \int_{-\infty}^{+\infty} \mathrm{d} x^{0}\left\langle\psi_{q_{f}}^{p}\left(x_{1}\right) \phi_{f}\left(x_{2}\right)\left|V_{d}\left(\boldsymbol{r}_{1}, \boldsymbol{r}_{2}\right)\right| \psi_{q_{i}}^{p}\left(x_{1}\right) \phi_{i}\left(x_{2}\right)\right\rangle,
\end{aligned}
$$

where $x_{1}=\left(c t, \boldsymbol{r}_{1}\right), x_{2}=\left(c t, \boldsymbol{r}_{2}\right)$ are, respectively, coordinates for positron and electron. The potential $V_{d}\left(r_{1}, r_{2}\right)=Z /\left|\boldsymbol{r}_{1}\right|-1 /\left|\boldsymbol{r}_{1}-\boldsymbol{r}_{2}\right|$ is the interaction between the unperturbed projectile (positron) and the target atom in the direct channel. We choose the target nucleus as the origin of the coordinate system which is assumed to be infinitely massive. The positions of incident positron and bound electron are labeled by $\boldsymbol{r}_{1}$ and 
$\boldsymbol{r}_{2}$, respectively. The wave functions $\psi_{q_{i}}^{p}\left(x_{1}\right)$ and $\psi_{q_{f}}^{p}\left(x_{1}\right)$ are the Dirac-Volkov states describing, respectively, the motion of the incident and scattered positrons, $\phi_{f}\left(x_{2}\right)$ is the Dirac-Volkov wave function of the ejected electron, while $\phi_{i}\left(x_{2}\right)$ describes the ground state of the hydrogen atom which is given by

$$
\phi_{i}\left(x_{2}\right)=\psi_{t}\left(t, r_{2}\right)=\exp ^{-\mathrm{i} \xi_{b} t} \psi_{t}\left(r_{2}\right),
$$

where $\psi_{t}\left(r_{2}\right)$ represents the hydrogenic relativistic function

$$
\begin{aligned}
& \psi_{t}\left(r_{2}\right)=\frac{(2 Z)^{\gamma_{H}+1 / 2}}{\sqrt{4 \pi}} \sqrt{\frac{\gamma_{H}+1}{2 \Gamma\left(2 \gamma_{H}+1\right)}} r_{2}^{\gamma_{H}-1} \mathrm{e}^{-Z r_{2}} \\
& \quad \times\left(\begin{array}{c}
1 \\
0 \\
\mathrm{i} \frac{\left(1-\gamma_{H}\right)}{Z \alpha} \cos (\theta) \\
\mathrm{i} \frac{\left(1-\gamma_{H}\right)}{Z \alpha} \sin (\theta) \mathrm{e}^{\mathrm{i} \phi},
\end{array}\right)
\end{aligned}
$$

where $Z$ is the atomic number, and the parameter $\gamma_{H}$ is

$$
\gamma_{H}=\sqrt{1-Z^{2} \alpha^{2}}
$$

and the binding energy of the ground state of atomic hydrogen is given by

$$
\xi_{b}=c^{2}\left(\sqrt{1-\alpha^{2}}-1\right),
$$

where $\alpha=1 / c$ is the fine structure constant.

\section{1. $R P W S G$}

To evaluate the TDCS in the absence of the laser field by the RPWSG, we first calculate the transition matrix element which gives the transition probability to the final state. The TDCS is a measure of probability that an $\left(\mathrm{e}^{+}\right.$, $\mathrm{e}^{+} \mathrm{e}^{-}$) reaction at an incident positron of energy $E_{i}$ and momentum $\boldsymbol{p}_{i}$, upon collision with the target produces scattered positron and ejected electron with energies $E_{f}$ and $E_{B}$ having momentum $\boldsymbol{p}_{f}$ and $\boldsymbol{p}_{B}$ and is defined, in atomic units, as

$$
\begin{aligned}
& \frac{\mathrm{d} \bar{\sigma}}{\mathrm{d} E_{B} \mathrm{~d} \Omega_{B} \mathrm{~d} \Omega_{i}}=\frac{\left|\boldsymbol{p}_{i}\right|\left|\boldsymbol{p}_{B}\right|}{2\left|\boldsymbol{p}_{f}\right| c^{6} \Delta^{4}} \\
& \times\left(\frac{1}{2} \sum_{s_{i}, s_{f}}\left|\bar{v}\left(p_{i}, s_{i}\right) \gamma^{0} v\left(p_{f}, s_{f}\right)\right|^{2}\right) \sum_{s_{B}}\left|\bar{u}\left(p_{B}, s_{B}\right) \gamma^{0}\right|^{2} \\
& \times\left|\Phi_{1,1 / 2,1 / 2}\left(\boldsymbol{\Delta}-\boldsymbol{p}_{B}\right)-\Phi_{1,1 / 2,1 / 2}\left(-\boldsymbol{p}_{B}\right)\right|^{2},
\end{aligned}
$$

where $\Omega_{i}$ and $\Omega_{B}$ are the emission solid angles of the positron and the ejected electron, respectively, $\Delta=$ $\boldsymbol{p}_{i}-\boldsymbol{p}_{f}$ is the momentum transfer vector, and functions $\Phi_{1,1 / 2,1 / 2}$ are the Fourier transforms of the relativistic atomic hydrogen wave functions. Using standard calculation in QED, spinorial parts reduce to traces in the Dirac algebra such that

$$
\begin{aligned}
& \sum_{s_{i}, s_{f}}\left|\bar{v}\left(p_{i}, s_{i}\right) \gamma^{0} v\left(p_{f}, s_{f}\right)\right|^{2}= \\
& \operatorname{Tr}\left(\gamma^{0}\left(c \not p_{f}-c^{2}\right) \gamma^{0}\left(c p_{i}-c^{2}\right)\right), \\
& \sum_{s_{B}}\left|\bar{u}\left(p_{B}, s_{B}\right) \gamma^{0}\right|^{2}=\operatorname{Tr}\left[\gamma^{0}\left(c p_{B}+c^{2}\right)\right],
\end{aligned}
$$

where $v(p, s)$ represents free positron bispinor which satisfies Dirac's equation for a free positron in the plane electromagnetic wave and which is normalized such that $\sum_{s_{i}} v\left(p_{i}, s_{i}\right) \bar{v}\left(p_{i}, s_{i}\right)=\not p_{i} c-c^{2}$.

\subsection{DVPWSG}

In the DVPWSG approach, we consider relativistic dressing of incident, scattered positrons and the ejected electron in which these particles can exchange one or more photons with the laser field.

The transition amplitude in the DVPWSG is given by $S_{f i}=\frac{i}{c} \int \mathrm{d} x^{0}\left\langle\psi_{q f}^{p}\left(x_{1}\right) \phi_{f}\left(x_{2}\right)\left|V_{d}\right| \psi_{q i}^{p}\left(x_{1}\right) \phi_{i}\left(x_{2}\right)\right\rangle$.

The wave functions $\psi_{q_{i}}^{p}, \psi_{q_{f}}^{p}$, and $\phi_{f}$ denote respectively the plane wave Volkov solution for the laser dressed incident, scattered positrons, and ejected electron given respectively by

$$
\psi_{q_{i}}^{p}\left(x_{1}\right)=\left[1-\frac{\not k A_{1}}{2 c\left(k \cdot p_{f}\right)}\right] \frac{v\left(p_{f}, s_{f}\right)}{\sqrt{2 Q_{f} V}} \exp \left(\mathrm{i} s_{f}\left(x_{1}\right)\right)
$$

and

$$
\psi_{q_{f}}^{p}\left(x_{1}\right)=\left[1-\frac{\not k A_{(1)}}{2 c\left(k \cdot p_{i}\right)}\right] \frac{v\left(p_{i}, s_{i}\right)}{\sqrt{2 Q_{i} V}} \exp \left(\mathrm{i} s_{i}\left(x_{1}\right)\right) .
$$

Here we have adopted the language of electrons on the left-hand side and the language of positrons on the right-hand side. An incoming electron with negative energy moving backward in time corresponds to an outgoing positron with positive energy moving forward in time. Here the Feynman slash notation is used, and $V$ is the normalization volume. Recall that $q=(Q / c, \boldsymbol{q})$ is the averaged four-momentum (dressed momentum) of the particle inside the laser field with vector potential $A$. Classically, the electromagnetic four potential, $A_{(1)}$, for a circularly polarized single mode laser of angular frequency $\omega$ can be expressed as

$$
A_{(1)}=a_{1} \cos \left(\phi_{1}\right)+a_{2} \sin \left(\phi_{1}\right),
$$

the phase of the laser field $\phi_{1}$ is as follows:

$$
\phi_{1}=k \cdot x_{1}=k_{0} \cdot x_{1}^{0}-\boldsymbol{k} \cdot \boldsymbol{x}_{1}=\omega t-\boldsymbol{k} \cdot \boldsymbol{x}_{1} .
$$

The phases $s_{i}\left(x_{1}\right)$ and $s_{f}\left(x_{1}\right)$ are given by

$$
\begin{aligned}
& s_{i}\left(x_{1}\right)=q_{i} \cdot x_{1}-\frac{\left(a_{1} \cdot p_{i}\right)}{c\left(k \cdot p_{i}\right)} \sin \left(\phi_{1}\right) \\
& +\frac{\left(a_{2} \cdot p_{i}\right)}{c\left(k \cdot p_{i}\right)} \cos \left(\phi_{1}\right), \\
& s_{f}\left(x_{1}\right)=q_{f} \cdot x_{1}-\frac{\left(a_{1} \cdot p_{f}\right)}{c\left(k \cdot p_{f}\right)} \sin \left(\phi_{1}\right) \\
& +\frac{\left(a_{2} \cdot p_{f}\right)}{c\left(k \cdot p_{f}\right)} \cos \left(\phi_{1}\right) .
\end{aligned}
$$

The ejected electron is modelled as follows:

$$
\begin{aligned}
& \phi_{f}\left(x_{2}\right)=\psi_{q_{B}}\left(x_{2}\right)= \\
& \quad\left[1+\frac{\not k A_{(2)}}{2 c\left(k \cdot p_{B}\right)}\right] \frac{u\left(p_{B}, s_{B}\right)}{\sqrt{2 Q_{B} V}} \exp ^{i s_{B}\left(x_{2}\right)},
\end{aligned}
$$

and the four-potential of the laser field felt by the ejected electron is given by 
with

$$
A_{(2)}=a_{1} \cos \left(\phi_{2}\right)+a_{2} \sin \left(\phi_{2}\right),
$$

$$
\phi_{2}=k \cdot x_{2}=k_{0} \cdot x_{2}^{0}-\boldsymbol{k} \cdot \boldsymbol{x}_{2}=\omega t-\boldsymbol{k} \cdot \boldsymbol{x}_{2},
$$

and the phases $s_{B}\left(x_{2}\right)$ is such as

$$
\begin{aligned}
& s_{B}\left(x_{2}\right)=-q_{B} \cdot x_{2}-\frac{a_{1} \cdot p_{B}}{c\left(k \cdot p_{B}\right)} \sin \left(\phi_{2}\right) \\
& +\frac{a_{2} \cdot p_{B}}{c\left(k \cdot p_{B}\right)} \cos \left(\phi_{2}\right) .
\end{aligned}
$$

In the same way as before, we get for the laser-assisted spin-unpolarized TDCS the following results:

$$
\begin{aligned}
& \frac{\mathrm{d} \bar{\sigma}}{\mathrm{d} E_{B} \mathrm{~d} \Omega_{B} \mathrm{~d} \Omega_{i}}= \\
& \left.\sum_{s, l=-\infty}^{+\infty} \frac{\mathrm{d} \bar{\sigma}^{(s, l)}}{\mathrm{d} E_{B} \mathrm{~d} \Omega_{B} \mathrm{~d} \Omega_{i}}\right|_{Q_{f}=Q_{i}+(s+l) \omega+\xi_{b}-Q_{B}},
\end{aligned}
$$

with

$$
\begin{aligned}
& \frac{\mathrm{d} \bar{\sigma}^{(s, l)}}{\mathrm{d} E_{B} \mathrm{~d} \Omega_{B} \mathrm{~d} \Omega_{i}}= \\
& \frac{1}{2} \frac{\left|\boldsymbol{q}_{i}\right|\left|\boldsymbol{q}_{B}\right|}{\left|\boldsymbol{q}_{f}\right| c^{6}} \frac{\sum_{s_{i} s_{f}}\left|M_{f i}^{(s)}\right|^{2} / 2}{\left|\boldsymbol{q}_{i}-\boldsymbol{q}_{f}+s \boldsymbol{k}\right|} \sum_{s_{B}}\left|\bar{u}\left(p_{B}, s_{B}\right) \Gamma^{l} \gamma^{0}\right|^{2} \\
& \times \mid \Phi_{1,1 / 2,1 / 2}\left(\boldsymbol{q}_{s, l}=\boldsymbol{q}_{i}-\boldsymbol{q}_{f}+(s+l) \boldsymbol{k}-\boldsymbol{p}_{B}\right) \\
& -\left.\Phi_{1,1 / 2,1 / 2}\left(-\boldsymbol{q}_{B}+l k\right)\right|^{2} .
\end{aligned}
$$

The sum $\sum_{s_{i} s_{f}}\left|M_{f i}^{(s)}\right|^{2} / 2$, which represents the positron spinorial contribution, has already been evaluated in a previous work [6]. The quantity $\sum_{s_{B}}\left|\bar{u}\left(p_{B}, s_{B}\right) \Gamma^{l} \gamma^{0}\right|^{2}$ which represents the sum over the spins of the ejected electron can be transformed to traces of the Dirac $\gamma$ matrices and evaluated using the soft REDUCE [23]:

$$
\begin{aligned}
& \sum_{s_{B}}\left|\bar{u}\left(p_{B}, s_{B}\right) \Gamma^{l} \gamma^{0}\right|^{2}= \\
& \quad 4\left\{E_{B} J_{l}^{2}\left(z_{B}\right)-\omega c\left(p_{B}\right)\left[\cos \left(\phi_{0 B}\right)\left(a_{1} \cdot p_{B}\right)\right.\right. \\
& \left.\quad+\sin \left(\phi_{0 B}\right)\left(a_{2} \cdot p_{B}\right)\right] J_{l}\left(z_{B}\right)\left[J_{l+1}\left(z_{B}\right)+J_{l-1}\left(z_{B}\right)\right] \\
& \left.\quad-a^{2} \omega\left(k \cdot p_{B}\right) c^{2}\left(p_{B}\right)\left[J_{l+1}^{2}\left(z_{B}\right)+J_{l-1}^{2}\left(z_{B}\right)\right]\right\}
\end{aligned}
$$

with

$$
\begin{aligned}
& c\left(p_{B}\right)=1 /\left[2 c\left(k \cdot p_{B}\right)\right], \\
& z_{B}=|a| \sqrt{\left(\hat{\boldsymbol{y}} \cdot \boldsymbol{p}_{B}\right)^{2}+\left(\hat{\boldsymbol{x}} \cdot \boldsymbol{p}_{B}\right)^{2}} / c\left(k \cdot p_{B}\right), \\
& \phi_{0 B}=\arctan \left[\left(\hat{\boldsymbol{y}} \cdot \boldsymbol{p}_{B}\right) /\left(\hat{\boldsymbol{x}} \cdot \boldsymbol{p}_{B}\right)\right],
\end{aligned}
$$

and the symbol $\Gamma^{l}$ is defined such as:

$$
\Gamma^{l}=C_{0_{B}} B_{l}\left(z_{B}\right)+C_{1_{B}} B_{1 l}\left(z_{B}\right)+C_{2_{B}} B_{2 l}\left(z_{B}\right) .
$$

The three quantities $C_{0_{B}}, C_{1_{B}}$, and $C_{2_{B}}$ are respectively given by

$$
\begin{aligned}
& C_{0_{B}}=1, \quad C_{1_{B}}=c\left(p_{B}\right) \phi_{1} \not k \\
& \text { and } C_{2_{B}}=c\left(p_{B}\right) \phi_{2} \not k .
\end{aligned}
$$

We compare this DVPWSG-TDCS with the corresponding cross-sections in the framework of the NRPWSG (where the incident, scattered, and ejected particles are described by non relativistic Volkov plane waves). The spin-unpolarized TDCS for NRPWSG is given by

$$
\begin{aligned}
& \frac{\mathrm{d} \bar{\sigma}^{\text {(NRPWSG) }}}{\mathrm{d} E_{B} \mathrm{~d} \Omega_{B} \mathrm{~d} \Omega_{i}}= \\
& \left.\quad \sum_{s, l=-\infty}^{+\infty} \frac{\mathrm{d} \bar{\sigma}^{(s, l)}}{\mathrm{d} E_{B} \mathrm{~d} \Omega_{B} \mathrm{~d} \Omega_{i}}\right|_{E_{f}=E_{i}+(s+l) \omega+\xi_{1 s}-E_{B}},
\end{aligned}
$$

with the expression of the TDCS accompanied by the transfer of $s+l$ number of photons given by

$$
\begin{aligned}
& \frac{\mathrm{d} \bar{\sigma}^{(s, l)}}{\mathrm{d} E_{B} \mathrm{~d} \Omega_{B} \mathrm{~d} \Omega_{i}}=\frac{2^{7}}{(2 \pi)^{2}} \frac{\left|\boldsymbol{p}_{i}\right|\left|\boldsymbol{p}_{B}\right|}{\left|\boldsymbol{p}_{f}\right|} \frac{J_{s}^{2}(z) J_{l}^{2}\left(z_{B}\right)}{\left|\boldsymbol{p}_{i}-\boldsymbol{p}_{f}+s \boldsymbol{k}\right|^{4}} \\
& \quad \times\left\{\frac{1}{\boldsymbol{q}_{s, l}^{2}+1}-\frac{1}{\left(\boldsymbol{p}_{B}-l \boldsymbol{k}\right)^{2}+1}\right\}^{2} .
\end{aligned}
$$

\section{Results and discussion}

In this paper, we present results for relativistic laserfree and laser-assisted positron-hydrogen atom scattering for various positron kinetic energies. The spinorial contribution is computed in closed analytic forms using the Software REDUCE [23], while the integral part, which represents the Fourier transforms of the relativistic atomic hydrogen wave functions, is also calculated analytically. The programming language FORTRAN and ROOT are used to plot the various figures of the present work.

\subsection{Process in the absence of laser field}

We consider the $\mathrm{e}^{+}$-atom scattering in the absence of the laser field. This may result in the elastic or inelastic scattering channels. In the following, we examine the ionization of the hydrogen atom in the ground state by positron impact. In addition, we assume that the energy of the projectile is sufficient to ionize the target leading to three unbound particles in the final state. Our aim is to show that, in the binary coplanar geometry and in the frame work of the first Born approximation, the present model is also able to predict TDCS at high energy in which the Coulomb effects are negligible. All the TDCSs are given in atomic units.

Figure 2 shows the summed laser-free TDCSs (DVPWSG ( $\epsilon=0$ a.u.), NRPWSG, and NRCBA [22]) for the ionization of hydrogen atom by positron impact. This figure represents the first check of our model in particular in the non-relativistic limit $\left(T_{i}=2700 \mathrm{eV}\right.$ and $\left.T_{f}=T_{B}=1349.5 \mathrm{eV}\right)$. In comparison between the three approaches, we find good agreement in particular for the binary coplanar geometry. This agreement gives us complete confidence in the numerical accuracy of our relativistic approximation and these tests allow to check the validity of DVPWSG model. We notice that there is 
a parfait symmetry around the value $\theta_{B}=45^{\circ}$ and a lobe of the binary peak appears in the vicinity of the same value. We will show in the coming figures that the magnitude of the binary peak decreases with increase of positron kinetic energy. This is admittedly a typical characteristic feature of the ionization of hydrogen atom by positron impact (for present work) or electron impact (see [15]). In this study, DVPWSG approach is mainly dealt with the Dirac-Volkov states. In the asymmetric coplanar geometry, the ejected electron produced in the initial binary collision, should undergo a recoil scattering from the ionized target in order to form the recoil peak on the same side of the incident positron (electron) beam. On the contrary in the symmetric coplanar geometry, the recoil peak for direct ionization of hydrogen atom by positrons impact was negligible in agreement with Vriens' model at large energy transfer [24].

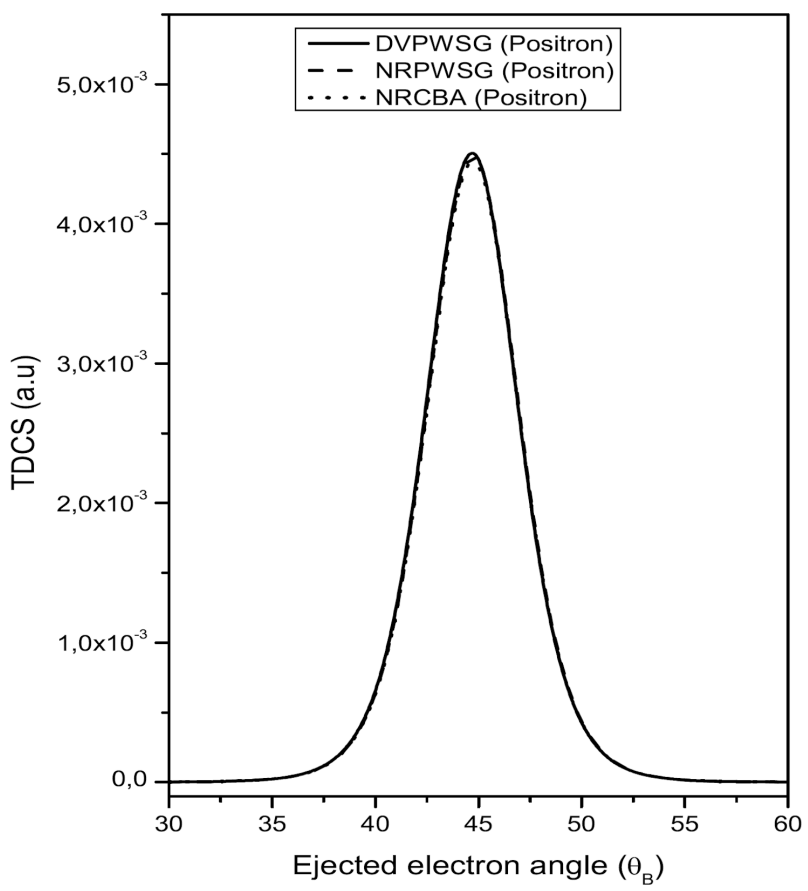

Fig. 2. The TDCS (in a.u.) as a function of the angle $\theta_{B}$ of the ejected electron. The incident kinetic energy of the positron is $T_{i}=2700 \mathrm{eV}$, the ejected electron energy is $T_{B}=1349.5 \mathrm{eV}$ and $\varepsilon=0$ a.u. [22].

Figure 3 shows a similar trend to that found in Fig. 2 for the two nonrelativistic models: the best agreement between the nonrelativistic Coulomb approximation and nonrelativistic plane wave Born approximation is found from the nonrelativistic limit $(2700 \mathrm{eV})$ and above. By increasing the energy, a different behavior appears: First, the binary peak position of the relativistic TDCS calculations is slightly shifted from the value $45^{\circ}$. Second, the agreement between the relativistic and nonrelativistic models is good from the nonrelativistic limit and below, but the discrepancies increase at high energies.

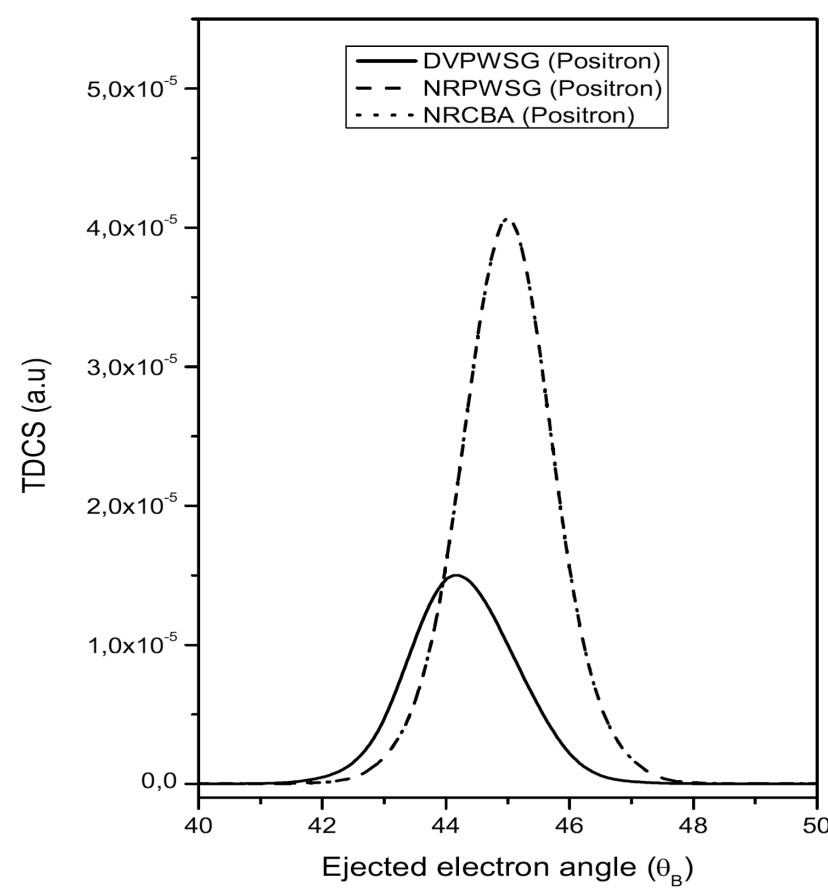

Fig. 3. The TDCS (in a.u.) as a function of the angle $\theta_{B}$ of the ejected electron. The incident kinetic energy of the positron is $T_{i}=51090 \mathrm{eV}$, the ejected electron energy is $T_{B}=25540.5 \mathrm{eV}$ and $\varepsilon=0$ a.u..

Figure 4 contains calculations made for the TDCS versus both positron energy and final angles $\theta_{B}$ in the binary coplanar geometry. For the variation with respect to $\theta_{B}$, we find that the TDCS decreases at small and large angles. Good agreement also exists between our calculations and several other works around the position of the binary peak. Only at the particular point of $\theta_{B}=\theta_{f}=45^{\circ}$ does the triple differential cross-section present a maximum, and the magnitude of the crosssection at this particular point decreases as the positron kinetic energy increases. The usual ionization process, responsible for the recoil peak in the coplanar asymmetric geometry, is almost excluded. However, as the incident positron energy increases the collision between the projectile and nucleus becomes weak, and the scattering angle of the projectile decreases, so that the double forward peaks, specific to the coplanar asymmetric geometry, should move together and become a single peak. At that time, the coplanar symmetric geometry still becomes the dominant one and the channel of the coplanar asymmetric geometry should be closed.

Figure 5 shows that when the incident positron kinetic energy increases under the condition $\theta_{B}=\theta_{f}=45^{\circ}$, the peak of the TDCS decreases and remains practically in the vicinity of $\phi_{B}=180^{\circ}$. From Fig. 5, we see that as the energy increases, the probability to observe positron in the direction $\phi_{B}=180^{\circ}$ diminishes gradually, which has also been found for electron impact ionization of hydrogen [25]. Figure 5 also shows that for all next figures in which we take $\phi_{B}$ to be constant, it must be equal to $180^{\circ}$ since the pick is clearly located at the same value $180^{\circ}$. 


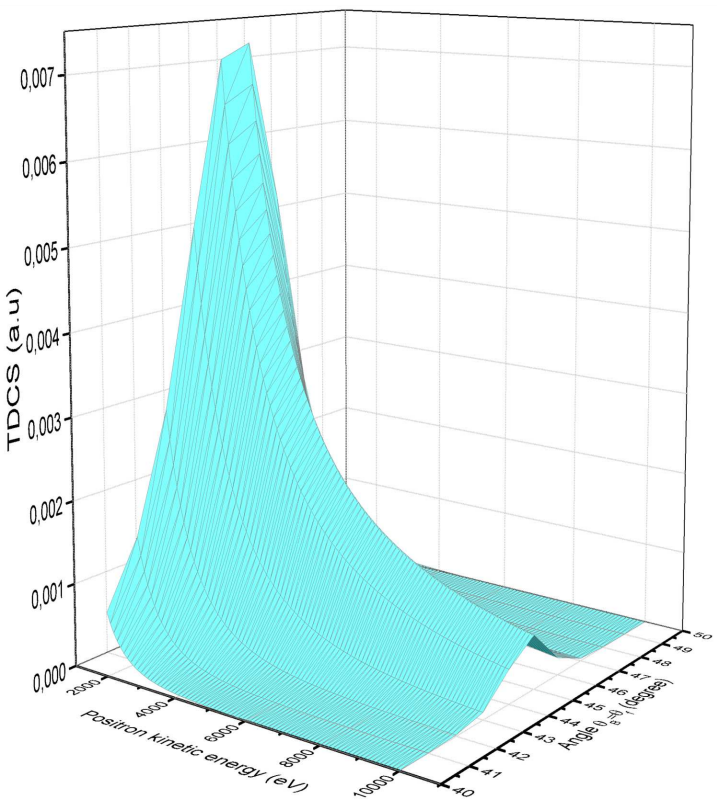

Fig. 4. The laser-free TDCS, in RPWSG, as a function of the angle $\theta_{B}$ of the ejected electron and the incident positron kinetic energy $T_{i}$ varying from $1000 \mathrm{eV}$ to $10,000 \mathrm{eV}$. We have used the condition $40^{\circ} \leq \theta_{f}=$ $\theta_{B} \leq 50^{\circ}$.

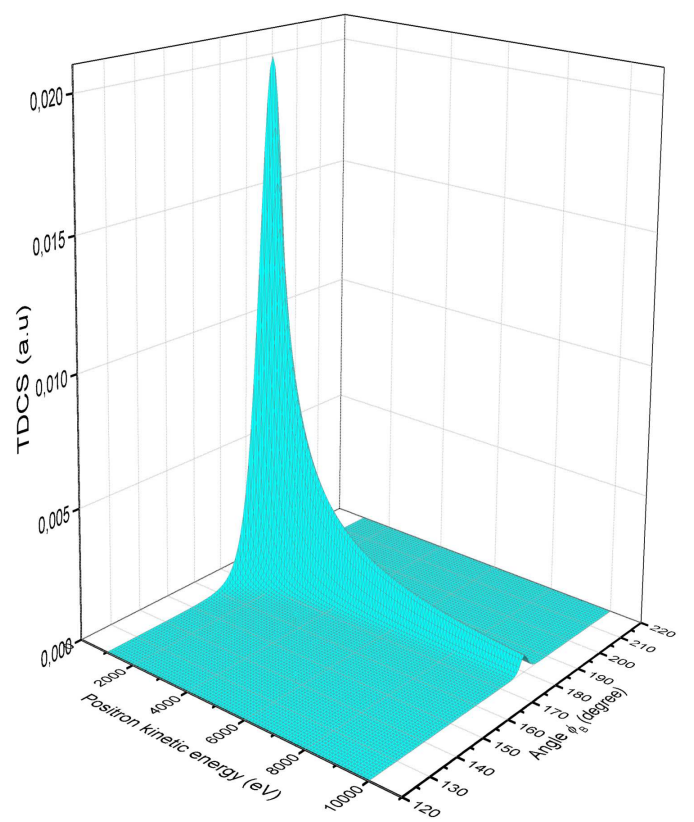

Fig. 5. The TDCS, in DVPWSG and NRPWSG, as a function of the angle $\phi_{B}$ of the ejected electron and the incident positron kinetic energy $T_{i}$ varying from $1000 \mathrm{eV}$ to $10,000 \mathrm{eV}$ for $\theta_{B}=\theta_{f}=45^{\circ}$.

It may be inferred that the coplanar asymmetric geometry is most prominent at low incident energies and for large nuclear charges. With nuclear charge such as $(Z=4,5)$, the forward peak appears in the cross-section for electron impact ionization of hydrogenic ions [26].
Here, we would like to emphasize that the collision processes proposed above are relevant within the use of relativistic approach, which can be studied either by laserfree TDCS using the Dirac formalism or by laser-assisted TDCS using the Dirac-Volkov treatment as it will be seen in the next section.

\subsection{Process in the presence of the laser field}

In the present investigation, the computations were performed for the geometry $\phi_{i}=\phi_{f}=0^{\circ}, \theta_{i}=0^{\circ}$, and $\theta_{f}=45^{\circ}$ for the incident and scattered positrons while for the ejected electron $\phi_{B}=180^{\circ}$ and the angle $\theta_{B}$ is a free parameter varing from $0^{\circ}$ to $180^{\circ}$.

Let us start our discussion of laser-assisted positronimpact relativistic ionization by illustrating and discussing the dressing effects of incident, scattered positrons and ejected electron by the external laser field on the triple differential cross-section. In order to illustrate the influence of the laser parameters (frequency and intensity) on the relativistic TDCSs, we shall present some typical results that we have obtained in the coplanar symmetric geometry. Figure 6 shows the dependence of the TDCS (DVPWSG) on the angular distribution of the ejected electron by varying the electric field strength. The most remarkable feature of the intensity dependence of TDCS is the fact that the magnitude of the TDCS decreases when the laser intensity increases. In Fig. 7, we present the TDCS as a function of the angle $\theta_{B}$ of the ejected electron for different frequencies of laser field. The numerical results show that the behavior of the TDCS strongly depends on the laser frequency. As much as the frequency increases, the TDCS continues to increase until the value $\omega=0.05$ a.u. and then the TDCS remains almost constant. It is also interesting to note that Figs. 6 and 7 reveal a qualitatively different behaviour of the TDCS as a function of the laser field intensity and frequency. Note that the ponderomotive potential energy is equal to the average kinetic energy of the oscillatory motion which is given, in atomic units, by

$$
U_{p}=\frac{e^{2} \epsilon^{2}}{4 m \omega^{2}}=\frac{I_{0}}{4 \omega^{2}} .
$$

Indeed, Newton's equation can be written simply as

$$
m \frac{\mathrm{d} v}{\mathrm{~d} t}=-e \epsilon \cos (\omega t)
$$

Assuming that the positron is at rest at $t=0$, we obtain

$$
v(t)=\frac{-e \epsilon}{m \omega} \sin (\omega t)
$$

For a particle experiencing harmonic motion, the timeaveraged kinetic reads

$$
\frac{1}{2} \overline{m v^{2}}=\frac{e^{2} \epsilon^{2}}{4 m \omega^{2}}=U_{p} .
$$

This is an additive quantity to the positron kinetic energy, so that an increase in intensity leads to a decrease in the TDCS (Fig. 6). Figure 7 depicts the reverse behavior than Fig. 6, an increase in frequency, TDCS increases until a such value in which the saturation occurs and then TDCS becomes insensitive to the frequency variation (Fig. 7). 


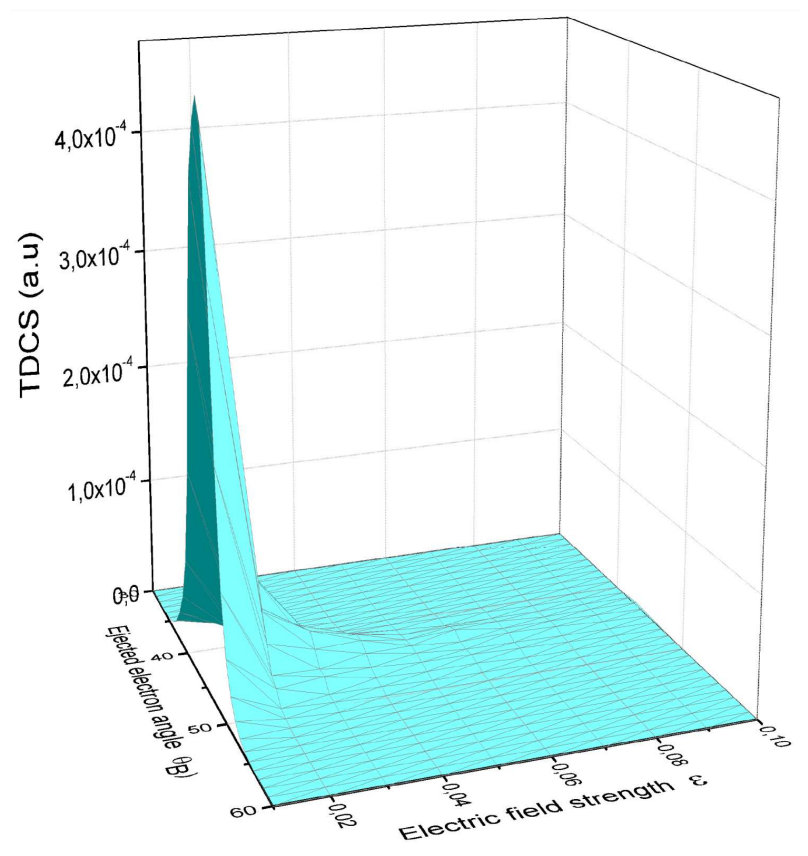

Fig. 6. The TDCS, in DVPWSG, as a function of the angle $\theta_{B}$ of the ejected electron and the electric field strength. The incident electron energy is $T_{i}=2700 \mathrm{eV}$, the ejected electron energy is $T_{B}=1349.5 \mathrm{eV}$. The frequency is $\omega=0.043$ a.u..

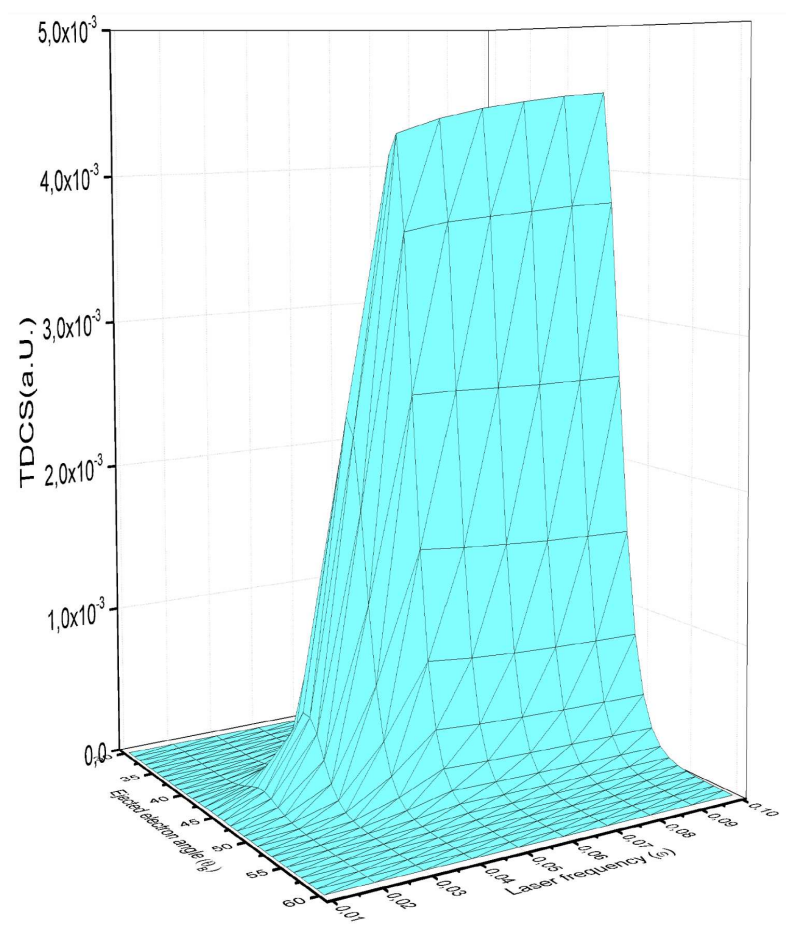

Fig. 7. The TDCS, in DVPWSG, as a function of the angle $\theta_{B}$ of the ejected and the laser frequency. The incident electron energy is $T_{i}=2700 \mathrm{eV}$, the ejected electron energy is $T_{B}=1349.5 \mathrm{eV}$. The electric field strength $\epsilon=0.01$ a.u..

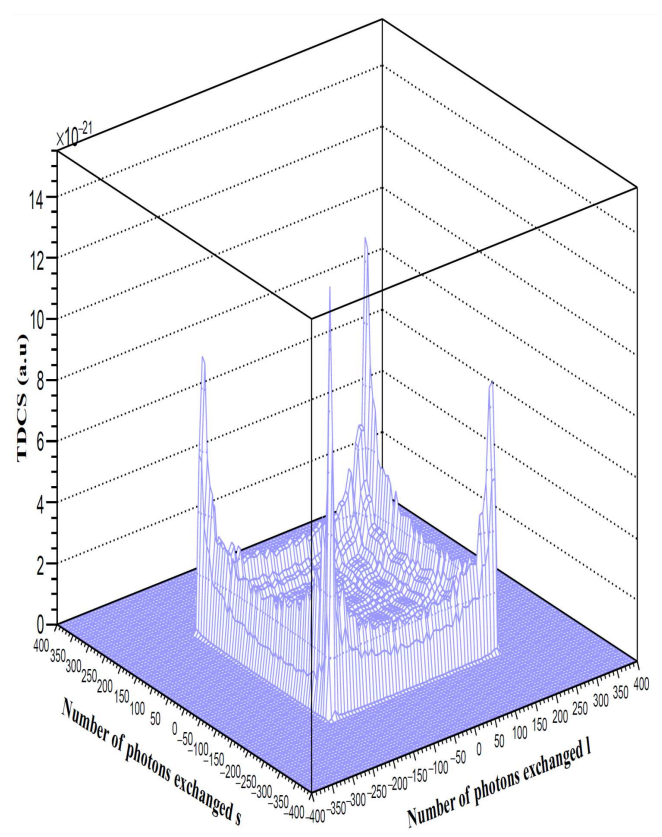

Fig. 8. The envelope of the TDCS, in DVPWSG, as function of the photon energy transfer in the nonrelativistic regime. The incident positron kinetic energy is $T_{i}=51090 \mathrm{eV}$, ejected electron kinetic energy $T_{B}=25540.5 \mathrm{eV}$, the electric field strength $\epsilon=0.01 \mathrm{a} . \mathrm{u}$. and the angle of the ejected electron is $\theta_{B}=45^{\circ}$.

The envelope of photon energy transfer obtained is a curve in three dimensions given in Fig. 8 in which we observe a rapid fall off of the relativistic TDCS for $s \simeq l \simeq \pm 200$ where the absolute value of the indices of the ordinary Bessel functions are close to their arguments. Also, as a side result, we see clearly in this figure that there is a complete symmetry between $s$ and $l$. To obtain a converging envelope, one has to sum over the same numbers $s$ and $l$ of photons exchanged. We now turn to the effects of the number of photons exchanged in laser-assisted TDCS. In Fig. 9, the famous Kroll-Watson (KW) sum rule [27] is found to be valid for incident positron kinetic energy $T_{i}=51090 \mathrm{eV}$ and ejected electron kinetic energy $T_{B}=25540.5 \mathrm{eV}$. From this figure and as a general comment, we observe that the magnitudes of the TDCSs increase when the number of the photons exchanged increases. By varying the number of photons exchanged, we have obtained the results that converge to the relativistic laser-free TDCS. In this case, one needs a number at least equal to \pm 200 photons to reach this convergency (the Kroll-Watson sum rule is satisfied for $s=l= \pm 200$ ). As for the quantitative laser modifications, the laser-assisted TDCSs tend to approach the laser-free results with the increasing number of photons exchanged indicating that the famous KW [27] sum rule which is mathematically modeled such as

$$
\sum_{s=-\infty}^{+\infty} \sum_{l=-\infty}^{+\infty} \frac{\mathrm{d} \bar{\sigma}^{(s, l)}}{\mathrm{d} E_{B} \mathrm{~d} \Omega_{B} \mathrm{~d} \Omega_{i}}=\frac{\mathrm{d} \bar{\sigma}^{\text {(laser-free) }}}{\mathrm{d} E_{B} \mathrm{~d} \Omega_{B} \mathrm{~d} \Omega_{i}},
$$

is valid for the present model. 


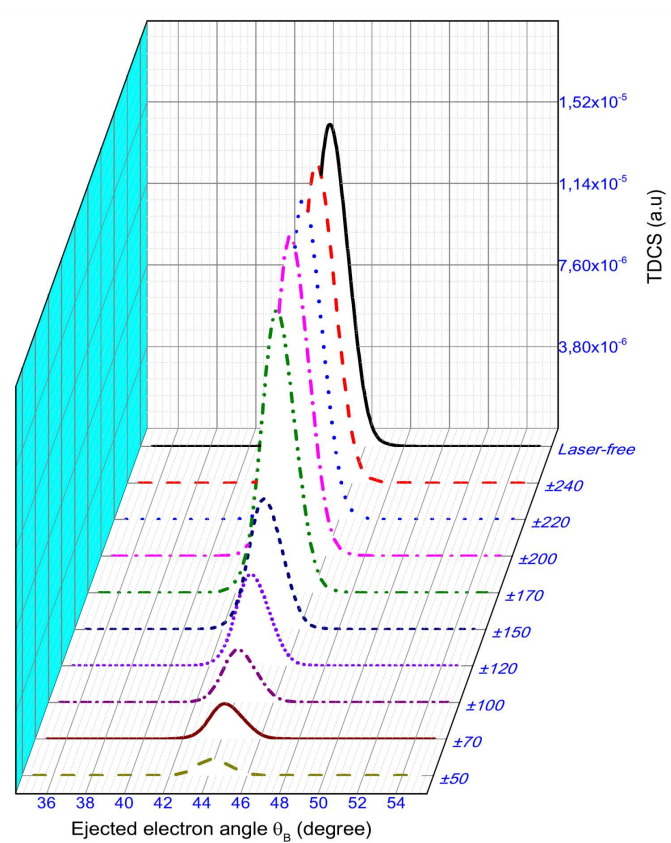

Fig. 9. The angle $\theta_{B}$ dependence of the TDCSs, in DVPWSG, with and without laser field for various sets of the numbers of photons exchanged. The incident positron kinetic energy is $T_{i}=51090 \mathrm{eV}$, ejected electron kinetic energy $T_{B}=25540.5 \mathrm{eV}$ and the electric field strength $\varepsilon=0.01$ a.u..

\section{Conclusions}

Ionization triple differential cross-sections for the laserassisted $\mathrm{e}^{+}-\mathrm{H}$ system have been studied theoretically over a wide range of relativistic kinetic energies in the coplanar symmetric geometry. The results presented here clearly demonstrate the usefulness of the Dirac-Volkov plane waves to describe relativistic particles in the perspective of interpreting the relativistic laser-assisted ionization of hydrogen atom by positron impact processes at high energies. The understanding of relativistic atomic collisions is still incomplete and under debate. This is mainly because until very recently there has been a great lack of accurate experimental data particularly at high energies. We believe that our results should encourage experimental researcher to perform such experiments for laser-assisted atomic collision.

\section{References}

[1] P. Ashley, G. Laricchia, M. Charlton, Hyperfine Interact. 89, 389 (1994).

[2] C.M. Surko, J.R. Danielson, G.F. Gribakinand, R.E. Continetti, New J. Phys. 14, 065004-1 (2012).
[3] S.E. Fayer, A. Loreti, S.L. Andersen, Á. Kövér, G. Laricchia, J. Phys. B At. Mol. Opt. Phys. 49, 1 (2016).

[4] Shu-Min Li, Chen Ji, Jian-Ge Zhou, H.J. Yin, Phys. Rev. A 47, 1197 (1993).

[5] Shu-Min Li, S. Yi, Yao-Yang Liu, Zi-Fang Zhou, C. Ji, Phys. Lett. A 203, 209 (1995).

[6] B. Manaut, S. Taj, M. El Idrissi, Can. J. Phys. 91, 696 (2013).

[7] A. Chattopadhyay, C. Sinha, Phys. Rev. A 72, 053406-1 (2005).

[8] S. Ghosh Deb, C. Sinha, J. Phys. Conf. Series 194, 112001 (2009).

[9] M.Y. Zheng, G. Qin, Shu-Min Li, Phys. Rev. A 82, 033425-1 (2010).

[10] P. Cavaliere, C. Lione, G. Ferrante, Can. J. Phys. 60, 605 (1982).

[11] J. Pan, S.M. Li, B. Jamal, Opt. Lett. 32, 585 (2007).

[12] L. Jun, S.M. Li, Chin. Phys. Lett. 27, 103401-1 (2010).

[13] S. Ghosh Deb, A. Biswas, C. Sinha, J. Phys. B At. Mol. Opt. Phys. 44, 215201-1 (2011).

[14] C. Sinha, A. Biswas, S. Ghosh Deb, J. Phys. Conf. Series 388, 112006 (2012).

[15] Y. Attaourti, S. Taj, Phys. Rev. A 69, 063411-1 (2004).

[16] S. Taj, B. Manaut, M. El Idrissi, Y. Attaourti, L. Oufni, Chin. J. Phys. 51, 945 (2013).

[17] R.P. Feynman, Phys. Rev. 76, 749 (1949).

[18] R. Boudet, Ann. Fondat. Louis de Broglie 38, 1 (2013) (in French).

[19] R.P. Feynman, Rev. Mod. Phys. 20, 367 (1948).

[20] R.P. Feynman, Phys. Rev. 76, 769 (1949).

[21] R.P. Feynman, Science 153, 699 (1966).

[22] A. Chahboune, B. Manaut, E. Hrour, S. Taj, Atoms 4, 1 (2016)

[23] A.G. Grozin, Using REDUCE in High Energy Physics, Cambridge University Press, Cambridge, England 1997.

[24] T. Vriens, Physica 45, (1969).

[25] J. Berakdar, J.S.J. Briggs, J. Phys. B At. Mol. Opt. Phys. 29, 2289 (1996).

[26] Q. Shi, Z. Chen, J. Chenand, K. Xu, J. Phys. B At. Mol. Opt. Phys. 30, 2859 (1997).

[27] N.M. Kroll, K.M. Watson, Phys. Rev. A 8, 804 (1973). 